\title{
DIFFERENTIABILITY OF MONOTONIC FUNCTIONS
}

BY

L. A. RUBEL (URBANA, ILL.)

cette équation. Reste à étudier le cas où il existe deux nombres $m_{0}$ et $n_{0}$ (où $m_{0}<n_{0}$ ) tels que $g_{m_{0}}(x)=g_{n_{0}}(x)$. Alors $g_{n_{0}}(x)=g_{n_{0}-m_{0}}\left[g_{m_{0}}(x)\right]$, d'où $g_{n_{0}-m_{0}}(x)=x$. On a ensuite $f\left[g_{n_{0}-m_{0}-1}(x)\right]=g_{n_{0}-m_{0}}(x)=x$. La fonction $f(x)$ est done inverse de $g_{n_{0}-m_{0-1}}(x)$ et par suite elle est univalente. Comme continue, elle est strictement monotone. Il existe done en vertu du corollaire qui précède une infinité de fonctions $g_{\epsilon} C_{\langle 0,1\rangle}$ satisfaisant à (1).

EXEMPLE. Soit $\eta \epsilon\langle 0,1\rangle$ un nombre irrationnel. Posons

$$
f(x)=\left\{\begin{array}{ccl}
x+\eta & \text { pour } & 0 \leqslant x \leqslant 1-\eta \\
x+\eta-1 & \text { pour } & 1-\eta<x<1 \\
0 & \text { pour } & x=1
\end{array}\right.
$$

La fonction $f$ transforme done l'intervalle $\langle 0,1\rangle$ en lui-même, mais elle est discontinue en deux points. Nous allons montrer que la seule fonction $g \in C_{\langle 0,1\rangle}$ qui satisfait à l'équation (1) est la fonction $g=e$. En posant $\xi_{0}=g\left(\xi_{0}\right)$, (1) entraîne en effet $f\left(\xi_{0}\right)=g\left[f\left(\xi_{0}\right)\right]$. Le point $\xi_{1}=f\left(\xi_{0}\right)$ est done un point fixe de la transformation $g$. En posant $\xi_{1}=f\left(\xi_{n-1}\right)$, on constate par récurrence que tous les nombres $\xi_{n}$ sont des points fixes de la transformation $g$. Or l'ensemble des nombres $\xi_{n}$ étant dense dans $\langle 0,1\rangle$, la fonction continue $g$, dont l'ensemble des points fixes est dense, est nécessairement la fonction-identité.

UNIVERSTTE DE ŁÓDŹ

Rę̧u par la Rédaction le 3. 8. 1962 
creasing function $F(y)$ defined on $[f(a), f(b)]$ such that $F^{\prime}(f(x))=x$ for each $x \in[a, b]$.

Geometrically, the construction of $F^{\prime}$ is evident. An analytical expression for $F$ is

$$
F(y)=\sup \{t: f(t) \leqslant y\} .
$$

It is obvious that $F$ is non-decreasing. Hence, to show that $F$ is continuous, it is enough to show that the range of $F$ is all of $[a, b]$. This follows from the trivially verified fact that $F(f(x))=x$.

Now let $f(x)$ be a non-decreasing, but possibly discontinuous function on $[a, b]$. We must show that $f^{\prime}(x)<+\infty$ exists almost everywhere. Without loss of generality, we may suppose that $f$ is strictly increasing, and satisfies, moreover, the condition

$$
f(y)-f(x) \geqslant y-x \quad \text { whenever } y \geqslant x,
$$

since we could otherwise consider $f(x)+x$. If $F(y)$ is the function of Lemma 2 , then by Lemma $1, F^{\prime}(y) \leqslant+\infty$ exists almost everywhere. We write

$$
\frac{f(y)-f(x)}{y-x}=\frac{f(y)-f(x)}{F(f(y))-F(f(x))}=\left(\frac{F(f(y))-F(f(x))}{f(y)-f(x)}\right)^{-1} .
$$

Thus, for every point of continuity of $f(x)$ such that $f(x)$ does not lie in the exceptional set $E_{f}$ where $F^{\prime}$ fails to exist, we see that $f^{\prime}(x) \leqslant+\infty$ exists. But the set of points of discontinuity of $f$ is countable at most. Furthermore, $f^{-1}\left(E_{f}\right)$ has measure zero, since if $I$ is any interval, then $f^{-1}(I)$ is a union of intervals of total length not exceeding the length of $I$, by (2). Hence $f^{\prime}(x) \leqslant+\infty$ exists almost everywhere.

To complete the proof, we need only show that the set $E_{\infty}$, of those $x$ for which $f^{\prime}(x)=+\infty$, has measure zero. Our method is close to one used by Lipinski [4]. For $C>0$, let $E_{C}$ be the set of those $x \in(a, b)$ for which there exist $s=s_{x}$ and $t=t_{x}$ with $s<x<t$ such that

$$
f(t)-f(s)>C(t-s)
$$

It is clear that $E_{O}$ is open, and hence the disjoint union of open intervals:

$$
E_{0}=\bigcup\left(a_{n}, b_{n}\right) .
$$

Let us denote by $\left[a_{n}^{\prime}, b_{n}^{\prime}\right]$ any closed sub-interval of $\left(a_{n}, b_{n}\right)$ such that

$$
b_{n}^{\prime}-a_{n}^{\prime}=\frac{1}{2}\left(b_{n}-a_{n}\right) \text {. }
$$

Now it is clear that $\left[a_{n}^{\prime}, b_{n}^{\prime}\right]$ is covered by the open intervals $\left(s_{x}, t_{x}\right)$ for $x \epsilon\left[a_{n}^{\prime}, b_{n}^{\prime}\right]$ and that $\left(s_{x}, t_{x}\right) \subset\left(a_{n}, b_{n}\right)$. By the Heine-Borel theorem, there is a finite subcovering, say $\left(s_{k}, t_{k}\right)$ where $k=1,2, \ldots, N$; and if we choose a subcovering with $N$ as small as possible, then each point of $U\left(s_{k}, t_{k}\right)$ lies in at most two of the intervals $\left(s_{k}, t_{k}\right)$, because given any three open intervals with a common point, some one is contained in the union of the other two.

Hence, using (4), we have

(7) $b_{n}^{\prime}-a_{n}^{\prime} \leqslant \sum\left(t_{k}-s_{k}\right) \leqslant C^{-1} \sum\left\{f\left(t_{k}\right)-f\left(s_{k}\right)\right\} \leqslant 2 C^{-1}\left\{f\left(b_{n}\right)-f\left(a_{n}\right)\right\}$,

and then by $(6)$ and $(7)$ we have

(8) $\sum\left(b_{n}-a_{n}\right) \leqslant 4 C^{-1} \sum\left\{f\left(b_{n}\right)-f\left(a_{n}\right)\right\} \leqslant 4 C^{-1}\{f(b)-f(a)\}$.

Since $E_{\infty}$ is contained in each $E_{C}$, it follows, on letting $C \rightarrow \infty$, that $\boldsymbol{E}_{\infty}$ has measure zero, and the proof is complete.

We now show briefly that if $f$ is a jump function, then $f^{\prime}=0$ almost everywhere. To say that $f$ is a jump function is to say that $f(x)=\sum f_{k}(x)$ where $f_{k}(x)=0$ for $x<\alpha_{k}, f_{k}(x)=S_{k}$ for $x>\alpha_{k}, 0 \leqslant f_{k}\left(\alpha_{k}\right) \leqslant S_{k}$, and $\sum S_{k}<\infty$. Choose $C>0$, then $\varepsilon>0$, and let $g=\sum^{\prime} f_{k}$, where $\Sigma^{\prime}$ is a sum over a finite set of indices $k$ such that $\sum S_{k}-\sum^{\prime} S_{k}<\varepsilon C$. Then $h=f-g$ is non-decreasing, $h(b)-h(a)<\varepsilon O$, and $h^{\prime}=f^{\prime}$ except on finitely many points. By (8), we see that $\left\{x: h^{\prime}(x) \geqslant C\right\}$ can be covered by open intervals whose total length is less than $4 \varepsilon$. Hence $\left\{x: f^{\prime}(x) \geqslant C\right\}$ has measure zero for each positive $C$. Since $\left\{x: f^{\prime}(x)>0\right\}=\bigcup\left\{x: f^{\prime}(x) \geqslant 2^{-n}\right\}$, the result is proved.

\section{REFERENCES}

[1] F. Riesz and B. Sz.-Nagy, Leçons d'Analyse Fonctionnelle, Third edition, Paris and Budapest 1955.

[2] J. von Neumann, Functional Operators, Volume 1, Princeton 1950.

[3] R. P. Boas, Jr., Differentiability of jump functions, Colloquium Mathematicum 8 (1961), p. 81-82.

[4] J. S. Lip iński, Une simple démonstration du théorème sur la dérivée d'une fonction de sauts, ibidem 8 (1961), p. 251-255.

UNIVERSTTX OF ILLINOIS,

GOLUMBIA UNIVERSTTY

Reçu par la Rédaction le 29.5.1962 